Article

\title{
Real-Time Flow Behavior of Hot Mix Asphalt (HMA) Compaction Based on Rheological Constitutive Theory
}

\author{
Guoping Qian ${ }^{1,2}$, Kaikai Hu ${ }^{1}$, Xiangbing Gong ${ }^{1, * \mathbb{D}}$, Ningyuan $\mathrm{Li}^{3}$ and Huanan $\mathrm{Yu}^{1}{ }^{1}$ \\ 1 School of Traffic and Transportation Engineering, Changsha University of Science \& Technology, \\ Changsha 410114, China; guopingqian@csust.edu.cn (G.Q.); kaikaihu@stu.csust.edu.cn (K.H.); \\ huanan.yu@csust.edu.cn (H.Y.) \\ 2 Key Laboratory of Special Environment Road Engineering of Hunan Province, Changsha University of \\ Science \& Technology, Changsha 410114, China \\ 3 Department of Civil and Environmental Engineering, University of Waterloo, Waterloo, N2L 3G1, Canada; \\ ningyuanli@hotmail.com \\ * Correspondence: xbgong@csust.edu.cn; Tel.: +86-1760-365-8650
}

Received: 23 April 2019; Accepted: 24 May 2019; Published: 27 May 2019

\begin{abstract}
Compaction is the most critical stage during pavement construction, but the real-time rheological behavior in the compaction process of hot mix asphalt has not received enough attention. Rheological properties directly reflect the of mixture performance, the intrinsic directly reflects the influencing factors of compaction, and the pavement compactness and service life. Therefore, it is important to interpret the rheological properties of the asphalt mixture during the compaction process. In this paper, the improved Nishihara model was used to study the viscoelastic-plastic properties of the hot mix asphalt in the compaction process. Firstly, the improved Nishihara model was briefly introduced. Subsequently, the stress and strain correlation curves are obtained by the MTS (Material Testing System) compaction test, and the strain-time curve is fitted to determine the model parameter values. Finally, the parameters are substituted into the constitutive equation to obtain the strain-time curve and compared it with the test curve. The results show that the improved Nishihara model effectively depicts the real time behavior of the asphalt mixture in the compaction progress. The viscos and plastic parameters present certain differences, which reflects that the gradation and temperature have certain influence on the compaction characteristics of the mixture.
\end{abstract}

Keywords: hot mix asphalt; Nishihara model; viscoelastic-plastic; compaction process

\section{Introduction}

The compaction of asphalt pavement affects the performance of pavement structure and service life. Rutting, friable, pothole, and other failures usually occur when the pavement layers are not well compacted [1-3]. One of the reasons for the poor compaction is that there are no complete theories for the real time rheological properties of asphalt mixture under high temperature and non-compaction conditions. Therefore, it is necessary to establish an actual mechanical model to evaluate the deformation of the asphalt mixture during the construction compaction process.

Asphalt mixture is a kind of thermo-viscoelastic-plastic material; the flow behavior of hot mix asphalt is concluded to be directly related to the rheological properties. Its total strain includes elastic strain, viscoelastic strain, plastic strain, and viscoplastic strain [4-6]. Its mechanical properties are related to stress state, temperature, and loading time. There has been a lot of research that is aimed at using the constitutive model to predict the permanent deformation of asphalt mixture [7-9]. Various time-dependent constitutive models have been proposed in the past few decades $[10,11]$. 
Giunta et al. [12] established a constitutive model that consisted of the Maxwell model and hardened viscoplastic element to simulate the time-dependent non-linear behavior of asphalt mixtures. The model can reflect the experimental characteristics of the material and match thermodynamic requirements. Masad et al. $[13,14]$ used the Perzyna viscoplastic model to describe the mechanical behavior of asphalt mixtures in constant strain rate compression tests. However, this model does not lack the ability to represent the recovery behavior during repeated loading-unloading cycles, because the viscoelastic model is not coupled to the viscoplastic model. Sun et al. [15] used the internal variable theory and the thermodynamic orthogonal principle to establish a continuous two-stage viscoelastic-viscoplastic constitutive model for simulate the aging characteristics of asphalt mixtures.

Other researchers have proposed some models to study the mechanical response of asphalt mixture under high temperature conditions. Huang et al. [16] proposed a temperature-dependent HiSS plastic model, which can reflect the nonlinear plasticity, temperature, and load rate of asphalt mixture. Darabi et al. [17] proposed a phenomenological rate-dependent hardening-relaxation model that was coupled with nonlinear Schapery viscoelastic and Perzyna viscoplastic models to accurately predict the permanent deformation of asphalt mixture under cyclic compression loads at high temperatures. However, they did not pay attention to radial strain, so the model could not accurately describe the three-dimensional mechanical properties of the asphalt mixtures. Pasetto et al. [18] established a viscoelastic-plastic constitutive model by viscoelastic elements and a slider that is connected by elastic springs in parallel with three Maxwell components to analyze the mechanical response and creep deformation ability of asphalt concrete at high temperatures. However, there is a lack of in-depth study regarding the creep mechanism of asphalt mixture and its relationship with viscoelastic response. Chen et al. [19] proposed a comprehensive constitutive model that is capable of capturing the main mechanical properties of asphalt materials based on the thermodynamic finite strain frame model, which can predict the complex time- and temperature-dependent response of asphalt materials during elongation and compression, and it successfully predicts the permanent deformation of the road.

The constitutive theory to predict the permanent deformation of asphalt mixture has been extensively studied and successfully used in practice. However, the rheological properties of the hot mix asphalt during compaction and the change law of viscoelastic-plastic parameters are seldom studied. Chen [20] established the viscoelastic-plastic constitutive relation of hot mix asphalt by Material Testing System (MTS) compaction test and SGC (Superpave Gyratory Compactor) gyratory compaction test, while using the basic principles and methods of viscoelastic plastic force. Liu [21] used the Bodnet-Partom model to simulate the rheological properties of asphalt mixture during compaction and simulated it with finite element software.

The above-mentioned models are complicated to solve the parameters; on the contrary, the Nishihara model is intuitive and theoretically simple, and it can clearly and comprehensively reflect the viscoelastic-plastic in the roller compaction. However, it does not consider the effects of nonlinearity and temperature stress. Therefore, based on the Nishihara model, this paper establishes a nonlinear Nishihara model to simulate the rheological behavior of the asphalt mixture during compaction. Through a MTS compaction test that was designed to simulate the construction compaction process, the rheological parameters were obtained, and the feasibility and rationality of the model were verified. The relation between compaction properties and model parameters was finally discussed based on the real-time parameter analysis.

\section{Test Materials and Methods}

This chapter describes the asphalt performance, mixture gradations, and MTS compaction test methods. The MTS compaction test is used to obtain the model parameters and verify the model. Figure 1 shows the flow chart of this article. 


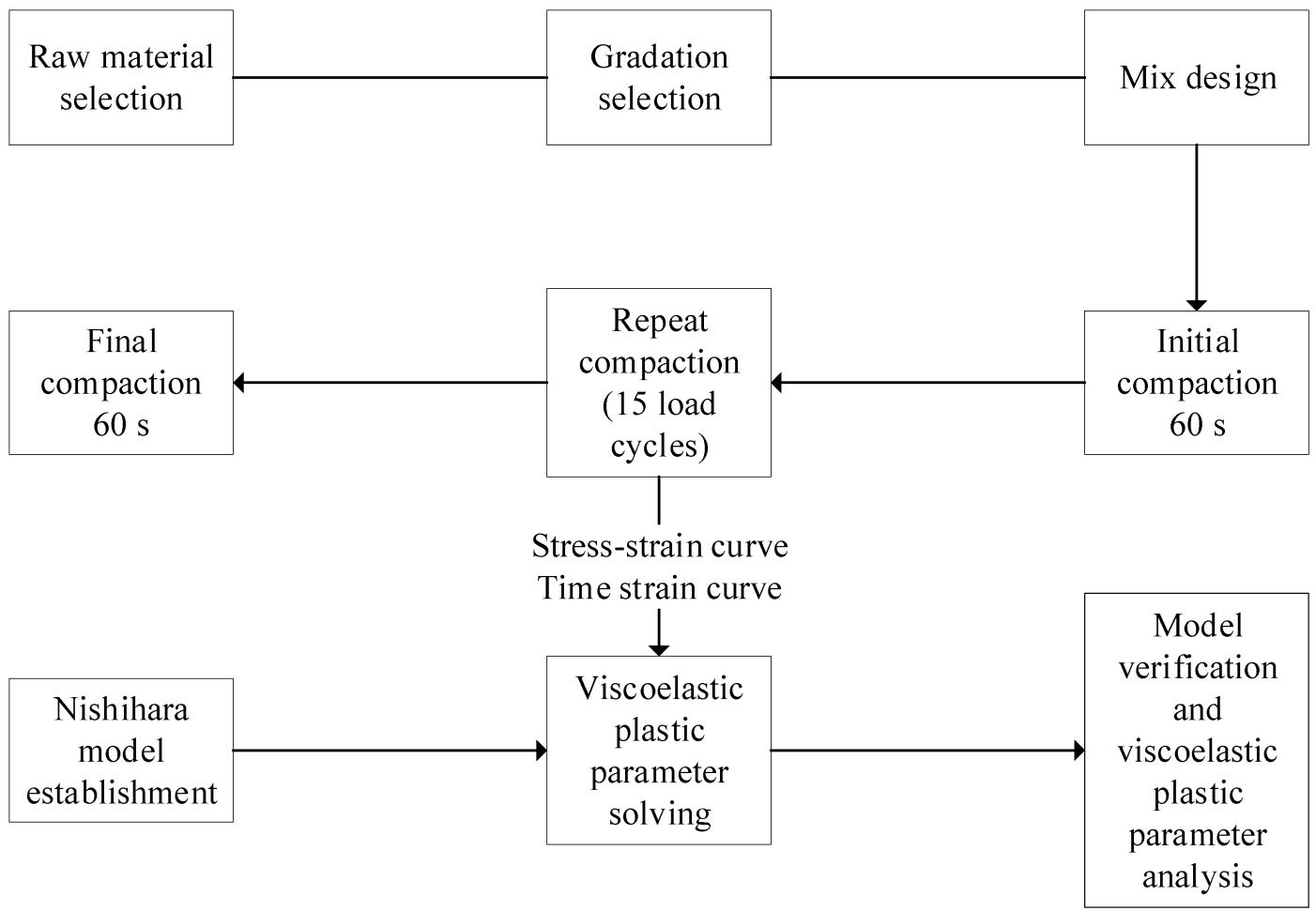

Figure 1. Flow chart of the materials and method used in this study.

\subsection{Materials and Mix Design}

The asphalt is a CNOOC Taizhou 70\# matrix asphalt, and Table 1 shows its physical properties. The aggregates used in this paper are basalt, and Tables 2 and 3 shows the physical properties. Aggregates with the size larger than $2.36 \mathrm{~mm}$ are coarse aggregates, and the aggregates with the size smaller than $2.36 \mathrm{~mm}$ are fine aggregates. The mineral filler is ground limestone, and Table 4 shows the physical properties. All of the tests are requirement Chinese standards [22-24].

Table 1. Physical Properties of 70\# matrix asphalt.

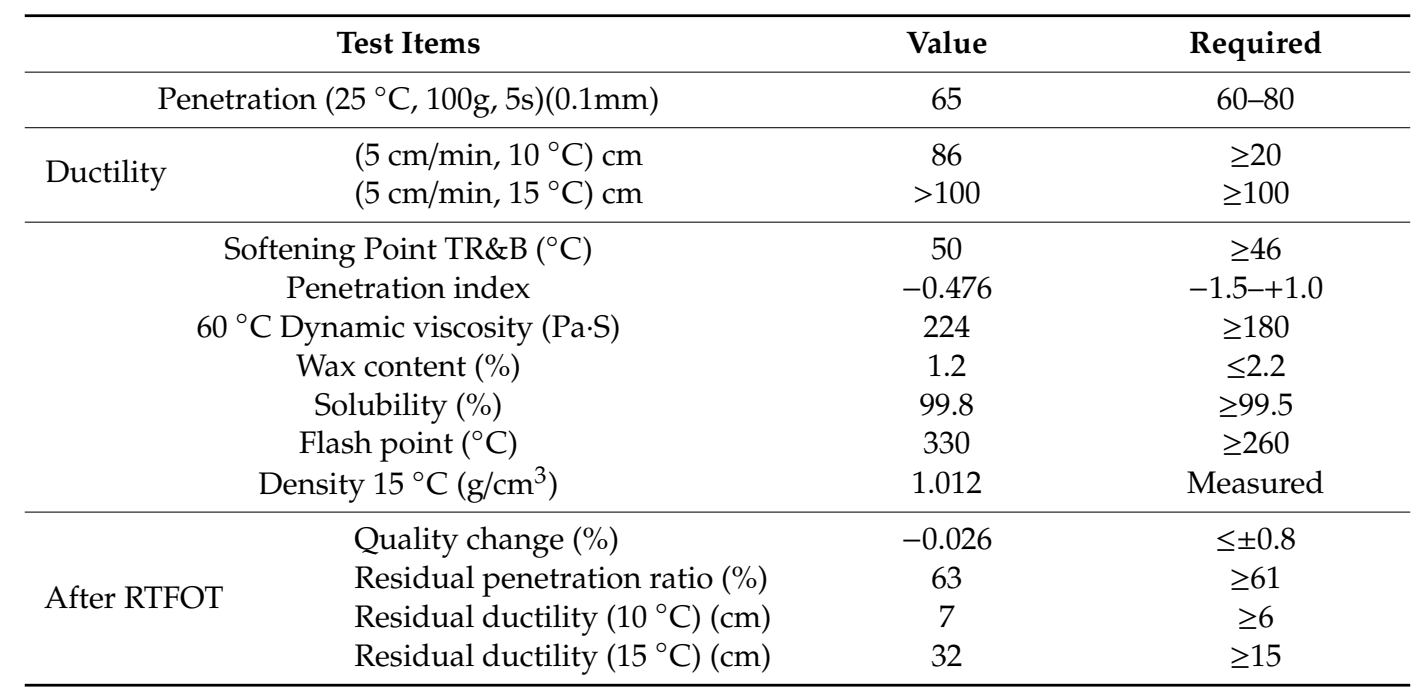


Table 2. Physical Properties of Coarse Aggregates.

\begin{tabular}{ccccc}
\hline \multirow{2}{*}{ Test Items } & \multicolumn{3}{c}{ Value } & Required \\
\cline { 2 - 4 } & $\mathbf{1 3 . 2 - 9 . 5}$ & $\mathbf{9 . 5 - 4 . 7 5}$ & $\mathbf{4 . 7 5 - 2 . 3 6}$ & \\
\hline Apparent relative density & 2.859 & 2.822 & 2.769 & $\geq 2.6$ \\
Water absorption (\%) & 0.52 & 0.57 & 0.73 & $\leq 2$ \\
Crushing value (\%) & 21 & - & - & $\leq 26$ \\
Los Angeles abrasion (\%) & 19.2 & - & - & $\leq 28$ \\
\hline
\end{tabular}

Table 3. Physical Properties of Fine Aggregates.

\begin{tabular}{ccc}
\hline Test Items & Value & Required \\
\hline Apparent relative density & 2.717 & $\geq 2.5$ \\
Mud content (percent of $<0.075 \mathrm{~mm})(\%)$ & 0.9 & $\leq 3$ \\
Sand equivalent $(\%)$ & 83.7 & $\geq 60$ \\
Angularity & 40.3 & $\geq 30$ \\
\hline
\end{tabular}

Table 4. Physical Properties of Mineral Filler.

\begin{tabular}{|c|c|c|c|}
\hline \multicolumn{2}{|c|}{ Test Items } & Value & Required \\
\hline \multicolumn{2}{|c|}{ Apparent relative density $\left(\mathrm{g} / \mathrm{cm}^{3}\right)$} & 2.638 & $\geq 2.5$ \\
\hline \multicolumn{2}{|c|}{ Water absorption $(\%)$} & 0.3 & $<1$ \\
\hline \multirow{3}{*}{ Grain sizes $(\%)$} & $<0.6 \mathrm{~mm}$ & 100 & 100 \\
\hline & $<0.15 \mathrm{~mm}$ & 99.9 & $90-100$ \\
\hline & $<0.075 \mathrm{~mm}$ & 90.2 & $75-100$ \\
\hline \multicolumn{2}{|c|}{ Hydrophilic coefficient } & 0.62 & $<1$ \\
\hline
\end{tabular}

Two different types of asphalt mixtures were used in the test: Fine grain asphalt concrete (AC-13) and multi-gravel asphalt concrete (SAC-13). Figure 2 shows the gradation. Multi-gravel asphalt concrete (SAC-13) contains more coarse aggregates, which can provide deeper surface structure, smaller voids and less water permeability, and good deformation resistance. The oil-stone ratios of AC-13 and SAC- 13 are $5.0 \%$ and $5.0 \%$, and the target air voids are $6.7 \%$ and $6.5 \%$, respectively.

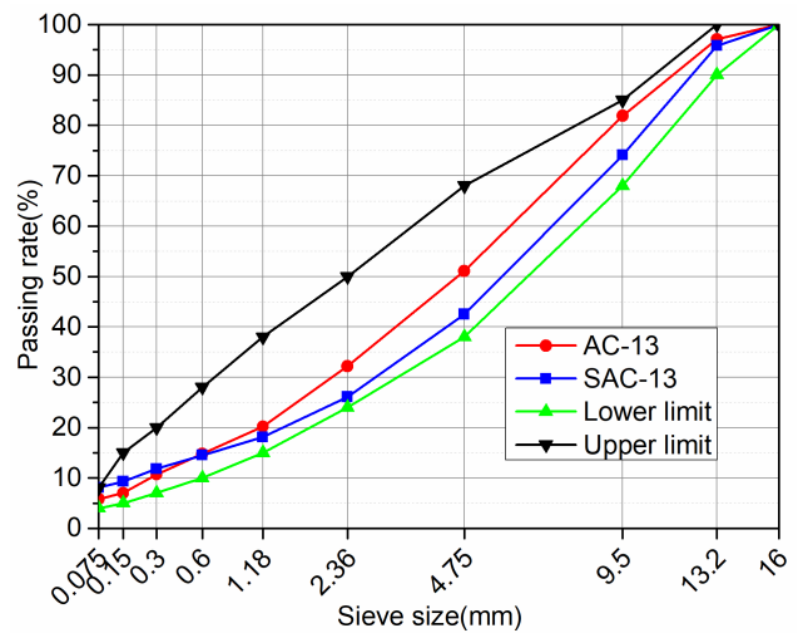

Figure 2. Gradation curves of AC-13 and SAC-13.

\subsection{MTS Compaction Process}

In order to get the complex parameters of the asphalt mixture compaction process and the change law of the temperature field, and to ensure the uniform distribution of aggregate. The test was carried 
out using a $48 \mathrm{~Hz}$ vibration platform, a Pt100 + paperless recorder, and a MTS810 material test system. The test plan mainly simulates pavement compaction. The asphalt mixture mixing temperature is $160{ }^{\circ} \mathrm{C}$ and the indoor ambient temperature is controlled to be about $25^{\circ} \mathrm{C}$. The mixed asphalt mixture was placed in a test piece cylinder having a diameter of $150 \mathrm{~mm}$ and a temperature sensor (Pt100) was buried in the mixture during the charging process. The mold containing the asphalt mixture was placed on a vibrating table for $10 \mathrm{~s}$, the vibration frequency was $48 \mathrm{~Hz}$, and then the mold was placed on the MTS for loading. Two parallel tests were performed on the same type of asphalt mixture.

To consider the rolling speed and the interval of the roller in a real construction project, Figure 3 shows the loading process. First compact for $60 \mathrm{~s}$ (initial compaction), then 15 cycles of loading (repeat compaction), and finally compact for $60 \mathrm{~s}$ (final compaction). The loading mode of cyclic load is: $1 \mathrm{~s}$ loading-2 s dead load-1 s unloading-2 s no load-1 s loading-2 s dead load-1 s unloading-30 s no load. Afterwards, repeat this cycle for 14 times. Figure 4 shows the first load cycle. Compaction load design considers SGC compaction load (generally 0.6 MPa) and relevant Chinese standards [23]. The initial and final compaction loads are $0.4 \mathrm{MPa}$, the repeated compaction loads are $0.6 \mathrm{MPa}$, and the total compaction time is $726 \mathrm{~s}$.

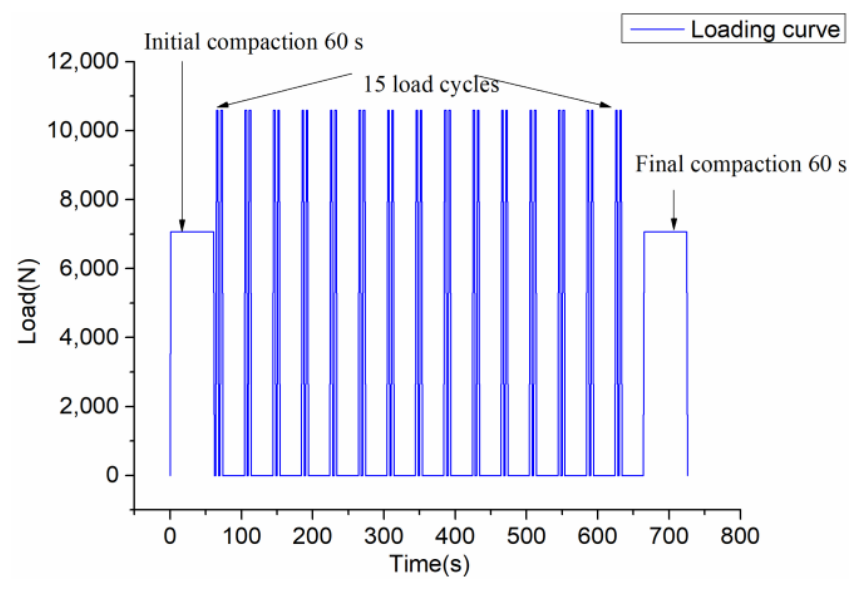

Figure 3. Loading curve and repeating cycles.

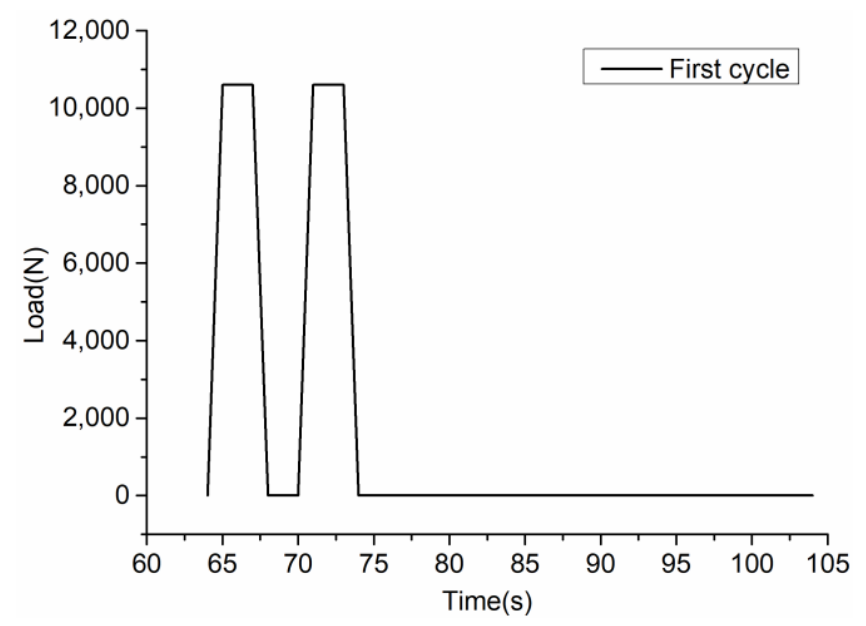

Figure 4. Loading curve of the first cycle.

The Pt100+ paperless recorder collects the temperature changes during the compaction process at a $1 \mathrm{~s}$ interval. The MTS computer is used to automatically record the pressure, time, and deformation during the test at a $0.005 \mathrm{~s}$ interval. 


\section{Viscoelastic-Plastic Rheological Model Theory}

\subsection{Nonlinear Viscoelastic-Plastic Nishihara Model and Parameter Solving}

The Nishihara model is widely used to study the rheological properties of rocks [25-30]. However, it does not consider the effects of nonlinearity and temperature stress. A nonlinear Nishihara model is established to analyze the rheological characteristics of materials based on the theory of viscoelastic-plastic Nishihara model. The nonlinear characteristic uses the power function type $\varepsilon(t)=A t^{k}$ ( $A$ is the test constant, $k$ is the rheological index. Its value depends on material properties, stress levels, and temperature conditions), as shown in Figure 5.

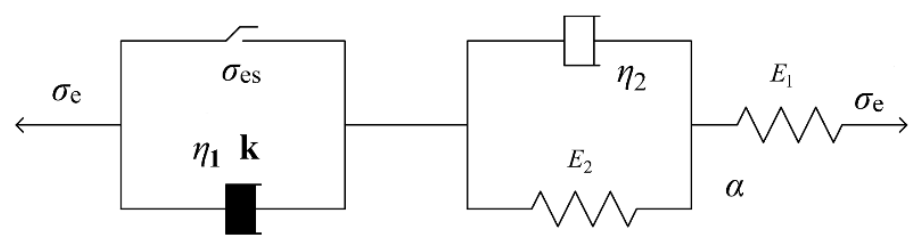

Figure 5. Nonlinear Nishihara model.

When considering the influence of temperature stress and temperature strain on the stress and strain of the material, the constitutive equation of the nonlinear Nishihara model can be written as:

$$
\varepsilon(t)=\left\{\begin{array}{lc}
\sigma_{e} / E_{1}+\sigma_{e} / E_{2}\left[1-\exp \left(-E_{2} t / \eta_{2}\right)\right]+\alpha \cdot \Delta T & \left(\sigma_{e}<\sigma_{e S}\right) \\
\sigma_{e} / E_{1}+\sigma_{e} / E_{2}\left[1-\exp \left(-E_{2} t / \eta_{2}\right)\right]+\left(\sigma_{e}-\sigma_{e S}\right) t^{k} / \eta_{1}+\alpha \cdot \Delta T & \left(\sigma_{e} \geq \sigma_{e S}\right)
\end{array}\right.
$$

where: $E_{1}$ is the elastic modulus of the Hooker body; $E_{2}$ is the elastic modulus of the Kelvin body; $\eta_{1}$, $\eta_{2}$ are the viscosity coefficients of the Kelvin body and the Bingham body stick component; $\sigma_{e}$ is the applied stress; $\sigma_{e S}$ is the material yield stress; $t$ is time; $k$ is the rheological index; $\Delta T$ is the calculation of the temperature difference; and, $\alpha$ is the material shrinkage coefficient.

The above formula fully considers the influence of factors, such as nonlinearity, time, and temperature stress of the material. Therefore, the model can more comprehensively reflect the nonlinear viscoelastic-plastic properties of the material.

The material is subjected to compressive stress and it takes a negative value, and the compaction of the hot mix asphalt mix is generally completed above $100{ }^{\circ} \mathrm{C}$, so the asphalt mixture is in a plastic flow dynamic, and the nonlinear viscous property is approximately linear viscous behavior, that is to take $k \approx 1$; at the same time, the temperature shrinkage stress $\sigma_{T}=E \alpha \cdot \Delta T \approx 0 \mathrm{MPa}$ (where $E=u \times 10^{3} \mathrm{MPa}, \Delta T \approx 0.1^{\circ} \mathrm{C}, \Delta T$ is the within 2 s loading temperature drop range). In addition, studies have shown that the higher temperature asphalt mixture has good stress relaxation performance, and the temperature stress that is generated by cooling can quickly relax to zero [31]. Therefore, the asphalt mixture pressure has no temperature stress in real time.

The constitutive equation of the nonlinear rheological model of the hot mix asphalt compaction process can be simplified as:

$$
\varepsilon(t)=-\left\{\sigma_{\mathcal{e}} / E_{1}+\sigma_{\mathcal{e}} / E_{2}\left[1-\exp \left(-E_{2} t / \eta_{2}\right)\right]+\left(\sigma_{\mathcal{e}}-\sigma_{e S}\right) t / \eta_{1}\right\} \quad\left(\sigma_{\mathcal{e}} \geq \sigma_{\mathcal{e S}}\right)
$$

where: $E_{1}$ is the elastic modulus of the Hooker body; $E_{2}$ is the elastic modulus of the Kelvin body; $\eta_{1}$, $\eta_{2}$ are the viscosity coefficients of the Kelvin body and the Bingham body stick component; $\sigma_{\mathcal{e}}$ is the applied stress; $\sigma_{e s}$ is the material yield stress; and, $t$ is time.

Equation (2) can ignore the influence of temperature effects during the calculation of stress and strain. It not only simplifies the model theory, but it also makes the determination of model parameters simpler and easier. The meaning of all parameters is shown in Table A1. 


\subsection{Model Parameter Solving}

In order to simulate the road compaction process, each load cycle includes two loading and unloading. It is assumed that the temperature is the same in one cycle, but different for different cycle. In Equation (2), there are six model parameters of $\sigma_{e}, \sigma_{e s}, E_{1}, E_{2}, \eta_{1}$, and $\eta_{2}$. Each parameter is a related parameter that varies with the number of load cycles and temperature.

In the analysis of viscoelastic-plastic parameters, the first loading process curve, the second unloading process curve, and the whole viscoplastic deformation in 15 loading cycles are analyzed. Combining the rheological model constitutive equation and the nonlinear regression equation, the solution ideas of each model parameter are presented, as follows:

(1) $\sigma_{e} \approx \sigma_{0}=0.6 \mathrm{Mpa}$.

(2) Under the action of 15 loading cycles, the value of $\sigma_{e s}$ in each cycle is during two loading and unloading processes. The tangent stress value at the straight line segment of the stress-strain curve at the first loading and the tangent point at the curve is taken as the $\sigma_{e s}$ of the cyclic process, as shown in Figure 6.

(3) Separating viscoplastic strain, elastic strain, and viscoelastic strain solves the parameters. Firstly, $E_{1}$ is solved by the "recovery elastic strain" after the second unloading in each cycle: $E_{1}=$ $\sigma_{e} / \varepsilon^{e}$. Secondly, the $\eta_{1}$ value is solved by the "median viscoplastic strain" in each cycle: $\eta_{1}=$ $2\left(\sigma_{e}-\sigma_{e S}\right) t / \varepsilon^{v p}(t)$. Thirdly, in the Kelvin body, $E_{2}$ and $\eta_{2}$ use the BoxLucas1 model equation $y=a\left(1-e^{-b x}\right)$ to perform nonlinear regression on the "recover viscoelastic strain time curve" after the second unloading in each cycle, and the parameters of the model are then obtained by calculating.

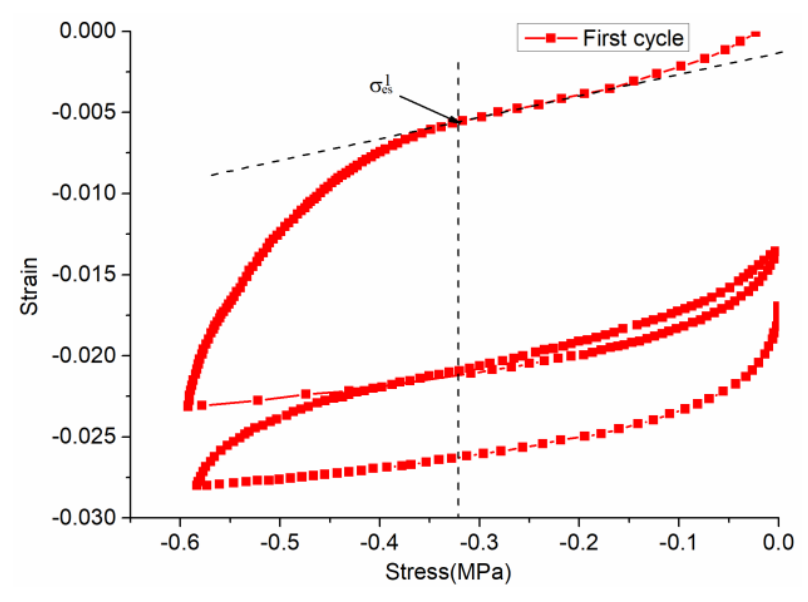

Figure 6. The first cyclic stress-strain curve of SAC-13.

The stress-strain-time response curves of the SAC-13 matrix asphalt mixture in the first cycle are taken as an example to solve the model parameters. Table 5 shows the viscoelastic plastic strain results of the SAC-13 asphalt mixture. 
Table 5. Viscoelastic-plastic strain of AC-13 and SAC-13 asphalt mixture.

\begin{tabular}{cccc}
\hline \multirow{2}{*}{ Noumber } & \multicolumn{3}{c}{ SAC-13 } \\
\cline { 2 - 4 } & Viscoplastic Strain $\left(\varepsilon^{v p}\right)$ & Elastic Strain $\left(\varepsilon^{e}\right)$ & Viscoelastic Strain $\left(\varepsilon^{v e}\right)$ \\
\hline First & -0.017470 & 0.007530 & 0.0034020 \\
Second & -0.008624 & 0.007504 & 0.0036927 \\
Third & -0.005866 & 0.007503 & 0.0038537 \\
Fourth & -0.004640 & 0.007496 & 0.0039342 \\
Fifth & -0.003839 & 0.007494 & 0.0041183 \\
Sixth & -0.003410 & 0.007491 & 0.0042349 \\
Seventh & -0.003276 & 0.007491 & 0.0040201 \\
Eighth & -0.002605 & 0.007439 & 0.0042196 \\
Ninth & -0.002484 & 0.007435 & 0.0041720 \\
Tenth & -0.002362 & 0.007421 & 0.0042173 \\
Eleventh & -0.002047 & 0.007420 & 0.0043321 \\
Twelfth & -0.001987 & 0.007418 & 0.0043233 \\
Thirteenth & -0.001820 & 0.007417 & 0.0044842 \\
Fourteenth & -0.001779 & 0.007401 & 0.0045524 \\
Fifteenth & -0.001740 & 0.007391 & 0.0043724 \\
\hline
\end{tabular}

The first step is to solve the yield stress $\sigma_{e s}$. Through the first cyclic stress-strain curve of Figure 6, the value of the tangent point at the straight line segment of the stress-strain curve at the first loading and the tangent point of the curve is determined, and $\sigma_{e s}^{1}$ is obtained. In the same way, $\sigma_{e s}^{2} \cdots \sigma_{e s}^{15}$ can be obtained. The second step is to solve $E_{1}, E_{2}, \eta_{1}$, and $\eta_{2}$. The elastic strain, viscoplastic strain, and viscoelastic strain have been marked in Figure 7, and the parameters of the table are solved, as follows:

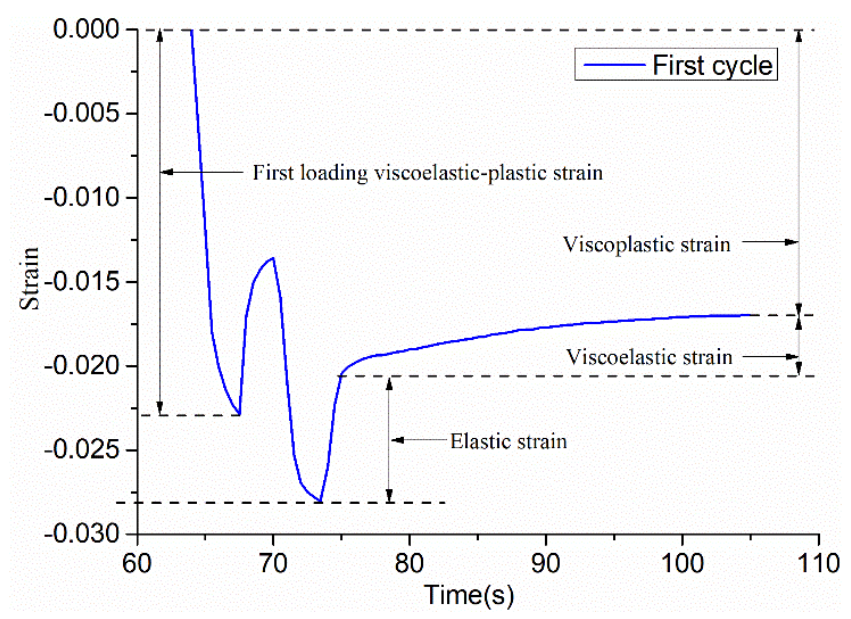

Figure 7. The first cycle strain-time curve of SAC-13.

(a) $E_{1}=\sigma_{e} / \varepsilon^{e}$, then $E_{1}^{1}=0.6 / \varepsilon^{e 1}=0.6 / 0.00753=79.681$, in the same way, $E_{1}^{2} \cdots E_{1}^{15}$ can be obtained.

(b) $\eta_{1}=2\left(\sigma_{e}-\sigma_{e S}\right) t / \varepsilon^{v p}(t)$, then $\eta_{1}^{1}=\left(\sigma_{e}-\sigma_{\mathrm{eS}}^{1}\right) \times 2 /\left(\varepsilon^{v p 1} / 2\right)$, that is $\eta_{1}^{1}=(0.6-0.340) \times$ $2 /(0.01747 / 2)=64.34$, and in the same way, $\eta_{1}^{2} \cdots \eta_{1}^{15}$ can be obtained.

(c) The BoxLucas 1 model equation $y=a\left(1-e^{-b x}\right)$ is used for non-linear regression, as shown in Figure 8. Where $a=\sigma_{e} / E_{2}, b=E_{2} / \eta_{2}$. After calculation, $E_{2}^{1}=0.6 / 0.00231=166.205$, $\eta_{2}^{1}=166.205 / 0.09950=1670.4$. In the same way, $E_{2}^{2} \cdots E_{2}^{15}$ and $\eta_{2}^{2} \cdots \eta_{2}^{15}$ can be obtained. 


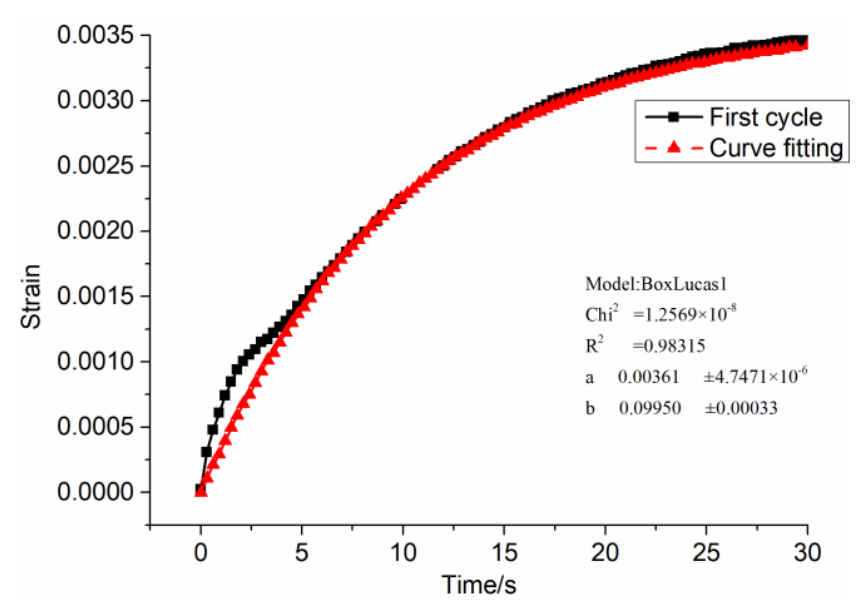

Figure 8. Nonlinear regression of the first cycle viscoelastic strain time curve of SAC-13.

\section{Nishihara Model Verification and Parameter Analysis}

\subsection{Model verification}

The six model parameters $\sigma_{e}, \sigma_{e s}, E_{1}, E_{2}, \eta_{1}$, and $\eta_{2}$ are substituted into Equation (2) to obtain the strain curves when compared with the testing strain-time curves, as shown in Figure 9.

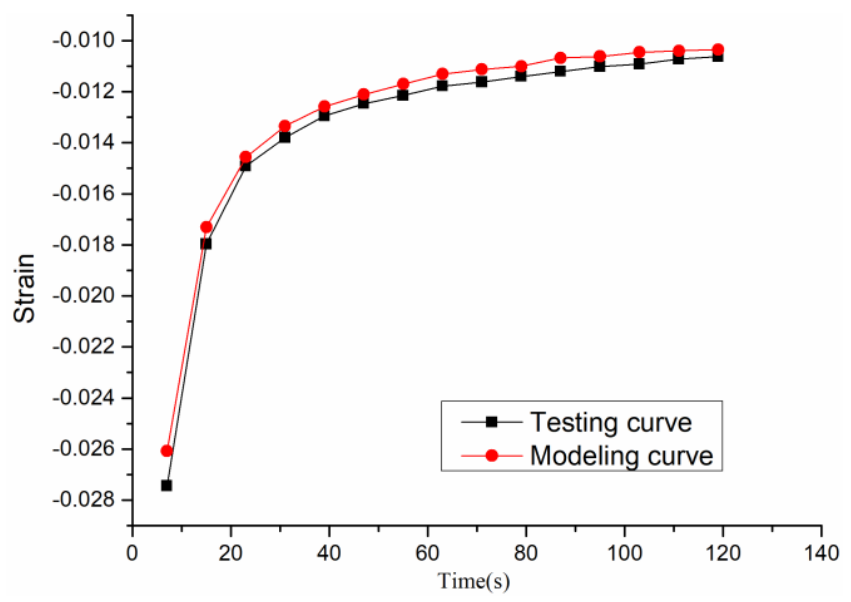

Figure 9. Testing curve and modeling curve in a Material Testing System (MTS) compaction test.

It can be seen that the flow before the third load cycle is obvious, because the early asphalt mixture is not compact enough. After the third load cycle, the deformation begins to decrease as the number of load cycles increases, because the asphalt mixture tends to be dense. It can be found the calculated value is slightly smaller than the experimental value, but the basic change law of the compression test can be simulated, and the difference between the two should be caused by the difference between the test pieces. This proves that the viscoelastic theory is used to simulate the rheological properties of hot mix asphalt in the compaction process with certain rationality and effectiveness.

\subsection{Parameter Analysis}

The model parameter values were calculated by nonlinear regression analysis of the response curve of the asphalt mixture. In this paper, Table 6 shows only the parameter values calculated for the fifteenth load cycle. 
Table 6. Parameters of the 15th load cycle.

\begin{tabular}{|c|c|c|c|c|c|c|c|c|}
\hline \multirow{3}{*}{ Mix type } & \multirow{3}{*}{$T\left({ }^{\circ} \mathrm{C}\right)$} & \multirow{3}{*}{$\begin{array}{c}\text { Air } \\
\text { Voids } \\
(\%)\end{array}$} & \multicolumn{6}{|c|}{ Value } \\
\hline & & & $\sigma_{e}$ & $\sigma_{e s}$ & $E_{1}$ & $E_{2}$ & $\eta_{1}$ & $\eta_{2}$ \\
\hline & & & (MPa) & (MPa) & (MPa) & (MPa) & $(\mathbf{P a} \cdot \mathbf{s})$ & $(\mathbf{P a} \cdot \mathbf{s})$ \\
\hline SAC & 124.5 & 6.7 & 0.6 & 0.513 & 81.18 & 115.83 & 200.041 & 1728.016 \\
\hline $\mathrm{AC}$ & 121.9 & 7.0 & 0.6 & 0.516 & 89.634 & 133.311 & 241.371 & 1877.990 \\
\hline
\end{tabular}

The viscoplastic viscous coefficient $\eta_{1}$ characterizes the viscous effect of the viscoplastic process of the mixture, which reflects the ability of the asphalt mixture to resist deformation during the viscoplastic phase. Figure 10 indicates that, as the number of loading cycle increases, the temperature of the mixture decreases, and the yield stress and viscosity coefficient increase. The viscosity coefficient mainly increases because the viscosity of the asphalt increased when the temperature decreases. The rate change of yield stress and viscosity coefficient of the real-time mixture is faster and the mixture is easily compacted. After the third load cycle, the rate of change of yield stress and viscosity coefficient of AC-13 began to decrease. After the fourth load cycle, the rate of change of yield stress and viscosity coefficient of SAC-13 also began to decrease, and the mixture began to become more difficult to compact.

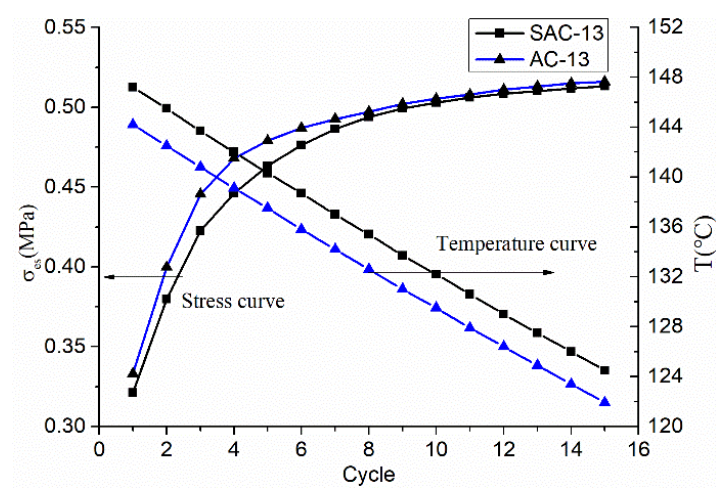

(a)

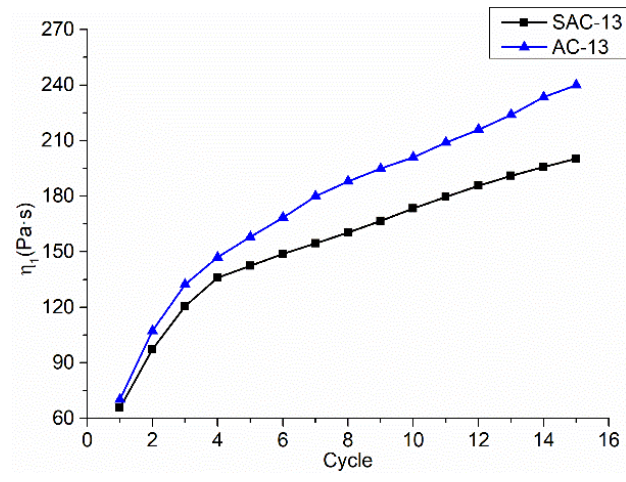

(b)

Figure 10. Model parameters and temperature curves with load cycle times; (a) $\sigma_{e s}$ and T curves with load cycle times; and, (b) $\eta_{1}$ curves with the load cycle times.

In Figure 11, as the number of load cycles increases, the air voids decreases. SAC-13 contains more coarse aggregates than AC-13 and the theoretical air voids should be relatively large. However, it can be seen that, as the number of load cycles increases, the air voids of SAC-13 begins to be less than AC-13. This shows that, the more coarse aggregate, the air voids is not necessarily greater, and the mixture is not necessarily harder to compact. The tendency of air void curves implies that AC-13 was becoming more difficult to be compacted.

$E_{1}$ is the elastic modulus, which is known as an index to measure the instantaneous difficulty of elastic deformation of materials. The larger the value, the greater the ability to resist deformation. Figure 12 presents that $E_{1}$ for SAC-13 stable as the number of load cycles changes, because the asphalt cement has strong fluidity at high temperature, and the elastic modulus of the mixture is mainly expressed as the elastic modulus of the aggregate skeleton in this state. The difference in the elastic modulus of the two grades is mainly due to the large amount of coarse aggregate and the high void ratio. 


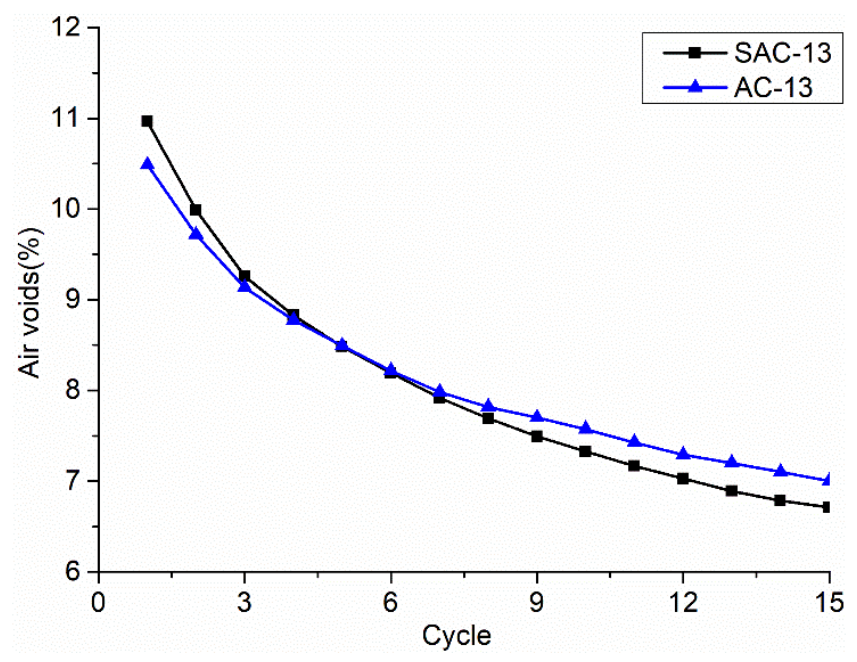

Figure 11. Air voids curves changing with the load cycle times.

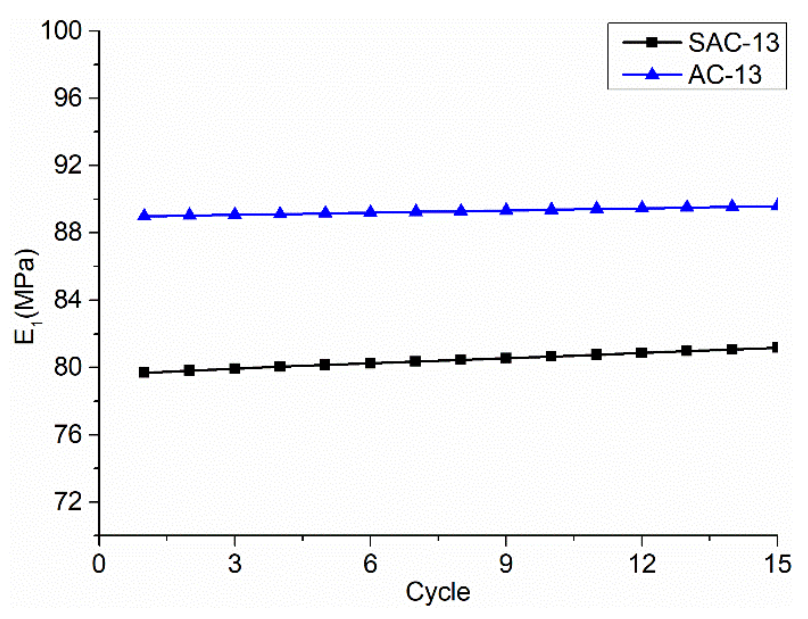

Figure 12. Elastic parameters curves changing with the load cycle times.

$E_{2}$ is the viscoelastic deformation modulus, which characterizes the viscoelastic recovery effect of the mixture. It can be seen from Figure 13 that, as the number of load cycles increases, $E_{2}$ decreases, and its ability to resist deformation decreases. The viscoelastic viscosity coefficient $\eta_{2}$ characterizes the viscous effect of the viscoelastic process of the mixture, which reflects the ability of the asphalt mixture to resist the deformation during the viscoelastic phase. Figure 13 presents that $\eta_{2}$ becomes larger as the number of load cycles increases, and the ability of the mixture to resist deformation becomes larger. The change in the two parameters is because, as the number of load cycles increase, the temperature decreases and the viscosity of the asphalt decreases. The difference in viscosity coefficients between the two grades is due to temperature differences and gradation differences, it can be summarized that the increasing rate of $\eta_{2}$ for AC-13 proves the difficulty in compaction. 


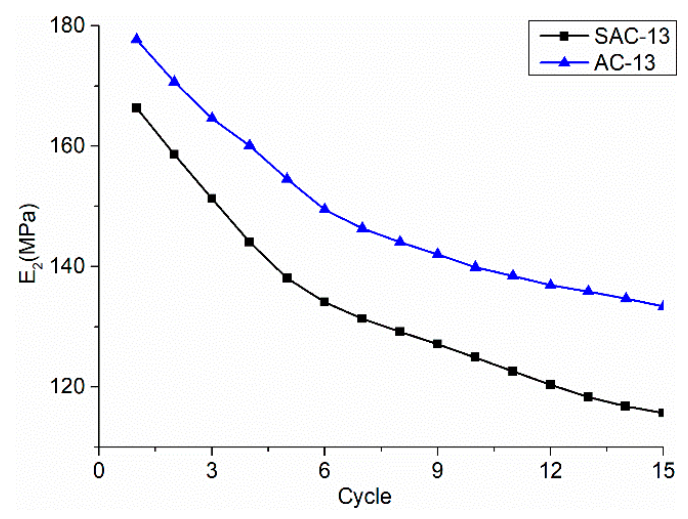

(a)

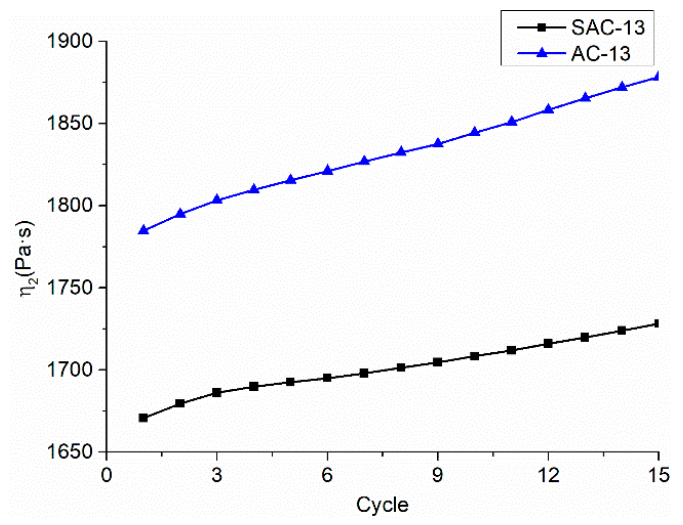

(b)

Figure 13. Viscoelastic parameters change with the load cycle times; (a) $E_{2}$ change with the load cycle times; and, (b) $\eta_{2}$ change with the load cycle times.

\section{Conclusion}

Based on the common Nishihara model, this paper establishes a constitutive equation for the nonlinear Nishihara model by considering the influence of nonlinearity and temperature stress of asphalt mixture. The MTS compaction test obtained the stress-strain curve and the time-strain curve, and the viscoelastic-plastic parameters were solved on this basis. Finally, the model parameters were substituted into the constitutive equation to verify, and the viscoelastic-plastic parameters of the two graded asphalt mixtures are analyzed.

1. The nonlinear Nishihara model has the advantages of being intuitive, simple in theory, and possessing easy to solve parameters. A loading and unloading MTS compaction test is effectively utilized to calculate the parameters. The calculation curve is compared with the test curve, and it is verified that the rheological properties of the hot mix asphalt in the compaction process can be indirectly characterized by the nonlinear Nishihara model.

2. The results prove that the nonlinear Nishihara model is useful in distinguishing the flow behavior undergoing a load and unloading compaction. The compaction of asphalt mixture mainly produces plastic deformation, which is mainly manifested by changes in viscos and plastic parameters $\left(\sigma_{e s}, \eta_{1}\right.$, and $\left.\eta_{2}\right)$, rather than the elastic parameter $\left(E_{1}\right)$. The differences in sensitive parameters $\left(\sigma_{e s}, \eta_{1}\right.$, and $\left.\eta_{2}\right)$ reflect that the gradation and temperature have certain influence on the compaction characteristics of the asphalt mixture.

3. Only two asphalt mixtures and one stress were included in this paper. The next step will be aiming at studies regarding the compaction properties subjected to various grades, different modified asphalts, and stress at different temperatures via the Nishihara model.

Author Contributions: G.Q. organized the research; K.H. performed compaction tests; G.Q. and X.G. wrote the manuscript; G.Q., X.G., N.L. and H.Y checked the manuscript.

Funding: The research was supported by National Key R\&D Program of the Ministry of Science and Technology of China (grant No.: 2018YFB1600100) and National Natural Science Foundation of China (grant No.: 51778071, and 51808058). The project was also supported by Open Fund of National Engineering Laboratory of Highway Maintenance Technology (Changsha University of Science \& Technology) (grant No: kfj180103).

Acknowledgments: This study was completed at the School of Traffic and Transportation Engineering, Changsha University of Science \& Technology.

Conflicts of Interest: The authors declare no conflict of interest. 


\section{Appendix A}

Table A1. Parameter comment table.

\begin{tabular}{|c|c|c|c|}
\hline$\sigma_{e}$ & Applied stress & $\varepsilon^{e}$ & Elastic strain \\
\hline$\sigma_{e s}$ & Yield stress & $\varepsilon^{v e}$ & Viscoelastic strain \\
\hline$E_{1}$ & The elastic modulus of the Hooker body & $k$ & The rheological index \\
\hline$E_{2}$ & The elastic modulus of the Kelvin body & $t$ & Time \\
\hline$\eta_{1}$ & $\begin{array}{l}\text { The viscosity coefficients of the Kelvin } \\
\text { body stick component }\end{array}$ & $\alpha$ & The material shrinkage coefficient \\
\hline$\eta_{2}$ & $\begin{array}{l}\text { The viscosity coefficients of the } \\
\text { Bingham body stick component }\end{array}$ & $\Delta T$ & The value of the temperature difference \\
\hline$\varepsilon^{v p}$ & Viscoplastic strain & & \\
\hline
\end{tabular}

\section{References}

1. Cui, W.; Tao, J.; Zhang, Z.; Li, H. Test on rut causes of expressway asphalt pavement. J. Chang'an Univ. 2009, $29,8-12$.

2. Zhang, K. Research on friable damage of asphalt pavement. Doctoral Dissertation, Chang'an University, Xi'an, China, 2015.

3. Liu, M.; Han, S.; Shang, W.; Qi, X.; Dong, S.; Zhang, Z. New polyurethane modified coating for maintenance of asphalt pavement potholes in winter-rainy condition. Prog. Org. Coat. 2019, 133, 368-375. [CrossRef]

4. Uzan, J.; Sides, A.; Perl, M. Viscoelastoplastic model for predicting performance of asphaltic mixtures. Transp. Res. Rec. 1985, 1043, 78-89.

5. Sides, A.; Uzan, J.; Perl, M. A comprehensive visco-elasto-plastic characterization of sand-asphalt under compression and tension cyclic loading. ASTM J. Test. Eval. 1985, 13, 49-59.

6. Li, J.; Zhang, J.; Qian, G.; Zheng, J.; Zhang, Y. Three-dimensional simulation of aggregate and asphalt mixture using parameterized shape and size gradation. J. Mater. Civil Eng. 2019, 31, 04019004. [CrossRef]

7. Cheng, Y.; Yu, D.; Tan, G.; Zhu, C. Low-temperature performance and damage constitutive model of eco-friendly basalt fiber-diatomite-modified msphalt mixture under freeze-thaw cycles. Materials 2018, 11, 2148. [CrossRef]

8. Sun, Y.; Gu, B.; Gao, L.; Li, L.; Guo, R.; Yue, Q.; Wang, J. Viscoelastic mechanical responses of HMAP under moving load. Materials 2018, 11, 2490. [CrossRef]

9. Cheng, Y.; Wang, W.; Tao, J.; Xu, M.; Xu, X.; Ma, G.; Wang, S. Influence analysis and optimization for aggregate morphological characteristics on high and low temperature viscoelasticity of asphalt mixtures. Materials 2018, 11, 2034. [CrossRef]

10. Lu, Y.; Wright, P.J. Temperature Related Visco-elastoplastic properties of asphalt mixtures. J. Transp. Eng. 2000, 126, 58-65. [CrossRef]

11. Lu, Y.; Lu, L.; Wright, P.J. Visco-elastoplastic method for pavement performance evaluation. Proc. Inst. Civil Eng.-Transp. 2002, 153, 227-234. [CrossRef]

12. Giunta, M.; Pisano, A.A. One-dimensional visco-elastoplastic constitutive model for asphalt concrete. Multidiscip. Model. Mater. Struct. 2006, 2, 247-264. [CrossRef]

13. Masad, E.; Tashman, L.; Little, D.; Zbib, H. Viscoplastic modeling of asphalt mixes with the effects of anisotropy, damage and aggregate characteristics. Mech. Mater. 2005, 37, 1242-1256. [CrossRef]

14. Masad, E.; Samer, D.; Little, D. Development of an elasto-viscoplastic microstructural-based continuum model to predict permanent deformation in hot mix asphalt. Int. J. Geomech. 2007, 7, 119-130. [CrossRef]

15. Sun, L.; Zhu, Y. A serial two-stage viscoelastic-viscoplastic constitutive model with thermodynamical consistency for characterizing time-dependent deformation behavior of asphalt concrete mixtures. Constr. Build. Mater. 2013, 40, 584-595. [CrossRef]

16. Huang, B.; Mohammad, L.N.; Wathugala, G.W. Application of a temperature dependent viscoplastic hierarchical single surface model for asphalt mixtures. J. Mater. Civ. Eng. 2004, 16, 147-154. [CrossRef]

17. Darabi, M.K.; Abu Al-Rub, R.K.; Masad, E.A.; Huang, C.-W.; Little, D.N. A modified viscoplastic model to predict the permanent deformation of asphaltic materials under cyclic-compression loading at high temperatures. Int. J. Plast. 2012, 35, 100-134. [CrossRef] 
18. Pasetto, M.; Baldo, N. Numerical visco-elastoplastic constitutive modelization of creep recovery tests on hot mix asphalt. J. Traffic Transp. Eng. 2016, 3, 390-397. [CrossRef]

19. Chen, F.; Balieu, R.; Kringos, N. Thermodynamics-based finite strain viscoelastic-viscoplastic model coupled with damage for asphalt material. Int. J. Solids Struct. 2017, 129, 61-73. [CrossRef]

20. Chen, X. The research on deformation characteristic of the HMA compaction. Master's Dissertation, Changsha University of Science \& Technology, Changsha, China, 2006.

21. Liu, Y. Visco-elastic-plastic model research on hot mix asphalt during the field compaction stage. Master's Dissertation, Changsha University of Science \& Technology, Changsha, China, 2009.

22. JTJ E20-2011. Standard Test methods of Bitumen and Bituminous Mixtures for Highway Engineering; Ministry of Communication: Beijing, China, 2011.

23. JTG F40-2004. Technical Specification for Construction of Highway Asphalt Pavements; Ministry of Communication: Beijing, China, 2004.

24. JTG E42-2005. The Methods of Aggregate for Highway Engineering; Ministry of Communication: Beijing, China, 2005.

25. Yan, Y.; Wang, S.-J.; Wang, E.-Z. Creep equation of variable parameters based on nishihara model. Yantu Lixue/Rock Soil Mech. 2010, 31, 3025-3035.

26. Pan, X.; Yang, Z.; Xu, J. Application study of nonstationary nishihara viscoelasto-plastic rheological model. Yanshilixue Yu Gongcheng Xuebao/Chinese J. Rock Mech. Eng. 2011, 30, 2640-2646.

27. Qi, Y.; Jiang, Q.; Wang, Z.; Zhou, C. 3D creep constitutive equation of modified nishihara model and its parameters identification. Yanshilixue Yu Gongcheng Xuebao/Chinese J. Rock Mech. Eng. 2012, 31, 347-355.

28. Jiang, Q.; Qi, Y.; Wang, Z.; Zhou, C. An extended nishihara model for the description of three stages of sandstone creep. Geophys. J. Int. 2013, 193, 841-854. [CrossRef]

29. Jiang, H.-F.; Liu, D.-Y.; Huang, W.; Xia, Y.-C.; Liu, F.-Y. Creep properties of rock under high confining pressure and different pore water pressures and a modified Nishihara model. Yantu Gongcheng Xuebao/Chinese J. Geotech. Eng. 2014, 36, 443-451.

30. Hou, F.; Lai, Y.; Liu, E.; Luo, H.; Liu, X. A creep constitutive model for frozen soils with different contents of coarse grains. Cold Reg. Sci. Technol. 2018, 145, 119-126. [CrossRef]

31. Tian, X.; Ying, R.; Zheng, J. Tests on thermal stress in asphalt cement sample and its calculation. China J. Highw. Transp. 2001, 14, 14-48. 